\section{Post-polio eradication: vaccination strategies and options for India}

\section{Jayakrishnan Thayyil, \\ Thejus Jayakrishnan ${ }^{2}$}

'Department of Community Medicine, Government Medical College, Calicut, India; 2Department of Surgical Oncology, Medical College of Wisconsin, Milwaukee, WI, USA

\section{Abstract}

In 1988, the World Health Organization (WHO) resolved to eradicate poliomyelitis globally. Since then, the initiative has reported dramatic progress in decreasing the incidence of poliomyelitis and limiting the geographical extent of transmission. 2013 is recorded as the second consecutive year not reporting wild poliovirus (WPV) from India. If the country can retain this position for one more year India will be declared as polio eradicated. What should be the future vaccination strategies? We searched and reviewed the full text of the available published literature on polio eradication via PubMed and examined Internet sources and websites of major international health agencies. The oral polio vaccine (OPV) has been the main tool in the polio eradication program. Once WPV transmission is interrupted, the poliomyelitis will be caused only by 0 PV. India could expect 1 vaccine-associated paralytic polio per 4.2-4.6 million doses of OPV. Considering the threat of vaccine-derived viruses to polio eradication, WHO urged to develop a strategy to safely discontinue OPV after certification. The ultimate aim is to stop OPV safely and effectively, and eventually substitute with inactivated polio vaccine (IPV). The argument against the use of IPV is its cost. From India, field based data were available on the efficacy of IPV, which was better than OPV. IPV given intradermally resulted in seroconversion rates similar to full-dose intramuscular vaccine. The incremental cost of adopting IPV to replace OPV is relatively low, about US \$1 per child per year, and most countries should be able to afford this additional cost

\section{Introduction}

2013 is recorded as the second consecutive year of not reporting wild poliovirus (WPV) from India. ${ }^{1}$ Operationally, eradication of polio is currently defined as the absence of a single indigenous case of acute flaccid paralysis (AFP) attributable to WPV in a defined geographical area for a period of three consecutive years. ${ }^{2}$ Hence, if this position can be retained for one more year, India will be declared polio-eradicated. The implicit promise of any eradication program is to end the intervention once the causative agent for the disease has been eradicated and apply the financial savings to other priority interventions. ${ }^{3}$ How do we prepare for posteradication of polio? What should be our vaccination strategies? We searched and reviewed the full text of the available published literature on polio eradication via PubMed and examined Internet sources and the websites of major international health agencies.

\section{Current status of wild poliovirus transmission: world}

In 1988, the World Health Organization resolved to eradicate poliomyelitis globally. Since then, the polio eradication initiative has reported dramatic progress in decreasing the incidence of poliomyelitis and limiting the geographical extent of transmission. ${ }^{1-3}$ The World Health Organization (WHO) Region of the Americas (1994), the Western Pacific Region (2000) and European Region (2002) have been certified as polio-free. ${ }^{4}$ The number of polio-endemic countries decreased from over 125 in 1988 to 7 in 2002 and 4 in $2008 .^{3}$ Until 2011, the wild polio endemic countries were confined to four AfroAsian countries referred to as PAIN (Pakistan, Afghanistan, India and Nigeria). India was removed from the list since January $2011 .^{5}$ During 2010-2011, environmental surveillance of WPV transmission was accomplished through testing of sewage samples in 21 countries without active poliovirus transmission. ${ }^{4}$ In 2009 twelve countries had circulating viruses and in 2010 four countries in the European Region (Kazakhstan, Tajikistan, Turkmenistan and the Russian Federation) experienced WPV outbreaks. During 2010-2011, 21 countries in the African, Eastern Mediterranean and South-East Asian Regions experienced WPV transmission. Re-established transmission continued in the previously polio-free countries of Angola, Chad and the Democratic Republic of the Congo, and WPV outbreaks occurred in 13 African countries and Nepal during 2010-2011. ${ }^{4}$ Twenty six countries have circulating WPV. ${ }^{4}$ In January 2012 , polio eradication was declared a programmatic emergency for global public health by the Executive Board of WHO. ${ }^{4}$

\section{Current status of wild poliovirus transmission: India}

Along with all 192 member nations of the WHO, the Government of India in 1988 com-
Correspondence: Jayakrishnan Thayyil, Department of Community Medicine, Government Medical College, 673008 Calicut, India.

E-mail: jayanjeeja@yahoo.co.in

Key words: wild poliovirus eradication, oral polio vaccine, inactivated polio vaccine, vaccinederived virus, vaccine-associated paralytic polio.

Received for publication: 12 October 2013.

Accepted for publication: 23 July 2014.

This work is licensed under a Creative Commons Attribution 3.0 License (by-nc 3.0).

(C) Copyright J. Thayyil and T. Jayakrishnan, 2014 Licensee PAGEPress, Italy

Healthcare in Low-resource Settings 2014; 2:1978 doi:10.4081/hls.2014.1978

mitted the nation to the goal of global polio eradication. Since 1995, the Ministry of Health and Family Welfare, Government of India has been conducting intensive immunization and surveillance activities aimed at the complete elimination of poliovirus and paralytic polio. ${ }^{1,6}$ In India polio cases decreased from 24,257 in 1988 to 4793 in 1994 with the help of routine immunization, well before the eradication program. ${ }^{7}$ The country has spent more than Rs 120 billion (US $\$ 2.5$ billion, US $\$ 1=$ Rs 50 ) on polio eradication after the program started in 1994, and Rs 1000 crore/year since 2000.5,8

India witnessed a surge of poliomyelitis type 1 in 2006. India is among the world's large reservoirs (63\%) of WPV, with 874 confirmed cases of poliomyelitis (wild virus) being reported in 2007 with 83 type 1 and 792 type 3 cases. ${ }^{6}$ During the following years the reported wild polio cases were 559 (2008), 741 (2009), 42 (2010), 1 (2011), 0 (2012). ${ }^{1}$ Historically, WPV transmission in India has mainly occurred in Bihar and Uttar Pradesh, two states with low coverage of routine immunization, migrant and remote subpopulations, and a lower relative effectiveness of oral poliovirus vaccine (OPV) compared with other areas. ${ }^{9}$

Combined with sensitive AFP surveillance, environmental surveillance has provided additional evidence to monitor the absence of WPV transmission in India. Sewage sampling was expanded from 10 sites in 2 states in 2010 to 15 sites in 4 states in $2011 .^{4}$ The last detected WPV from sewage testing in India was in November 2010 proving that there were no environmental transmissions. ${ }^{3}$ Keeping the country free of polio for two years was a feat that is a tribute to the Government of India and its 2.3 million vaccinators, who visited over 200 million households to ensure that the nearly 170 million children (under five years in age) were repeatedly immunised with $\mathrm{OPV}^{5}$ Now polio eradication in India is at a cross- 
road. We achieved this with the precious resources, enthusiasm of millions of health workers, commitment of governments and faith of hundreds of polio experts all over the globe. ${ }^{10}$ The absence of polio is both a measure of and means of development. ${ }^{8}$ So, the sustainability of this achievement is important.

\section{Problems after eradication with the use of oral poliovirus vaccine}

The live attenuated strains used in the OPV have been the main tool in the WHO polio eradication program. ${ }^{11}$ Like other developing countries in the national program we used OPV. However, these strains replicate in the human gut and are excreted for several weeks after immunisation. During this period, the attenuating mutations in the vaccine strains can rapidly revert. ${ }^{11}$ Once WPV transmission has been interrupted, poliomyelitis due to poliovirus will be caused only by $\mathrm{OPV} .^{12}$ Poliovirus isolates originating from OPV are, by definition, vaccine-derived polioviruses (VDPVs). ${ }^{3}$ They can cause vaccine-associated paralytic polio (VAPP) among recipients of vaccines, or their contacts, which can be subdivided into three: i) immunodeficient related excretors (iVDPVs) isolated from patients with congenital immunodeficiency syndrome who become chronically infected after exposure to OPV; ii) circulating VDPVs (cVDPVs) that arise and circulate in communities with low population immunity; and iii) ambiguous/other (aVDPVs) detected from healthy children or from environmental samples. ${ }^{3}$

Continued use of the OPV would, rarely, lead to prolonged excretion ( $>6$ months) of iVDPV from a person with a severe primary immunodeficiency syndrome. ${ }^{2}$ In 40 years of OPV use, 28 iVDPVs were documented by the end of 2004 . $^{2}$ In a hospital-based study among patients with primary immunodeficiency disorders in Sreelanka, it has been found that $10.2 \%$ of patients excreted poliovirus. ${ }^{13}$

Reports of cVPDPs were available from polio-eradicated countries. During 2000-2002, three outbreaks of cVDPVs were reported from Hispaniola, Madagascar, and the Philippines. ${ }^{14-}$ ${ }^{16}$ The attack rate and severity of disease associated with the recent cVDPV identified in Nigeria, a polio endemic country, were similar to those associated with WPV: ${ }^{17}$ they are genetically unstable Sabin-strain viruses that revert toward the genotypic and phenotypic profile of the virulent parent strain. ${ }^{18}$ An international review reported re-emergence of WPV in 21 previously polio-free countries. ${ }^{19}$ Within the last two year period, Angola, Chad, the Democratic Republic of Congo and Sudan have had year-long outbreaks. ${ }^{5}$ Hence, international planning for the management of the risk of WPV, after eradication, must include scenarios in which equally virulent and pathogenic cVDPVs could emerge. This should also be applicable to India. Outbreaks of poliomyelitis caused by VDPV have recently occurred in communities with long-term incomplete immunisation coverage. ${ }^{11}$ These chances are high in India where overall coverage of universal immunization program (UIP) was about $50 \%$, with low performing states like Bihar, and Uttar Pradesh below this level. As long as OPV is used, cVDPV and iVDPV pose a risk of causing poliomyelitis in unprotected individuals and threaten the goal of poliovirus eradication. ${ }^{11}$ In the case of reemergence of cVDPV similar to WPV with a potential of outbreak after OPV use, experts warn about the fragility of achievement of eradication with the current vaccination strategy and force us to accept the reality that we are fighting fire with fire. ${ }^{18}$ This paradox provides a major incentive for eventually stopping polio immunization or replacing OPV, but it also introduces complexity into the process of identifying safe and scientifically sound strategies for doing so. The core posteradication immunization issues include the risk/benefits of continued OPV use, the extent of OPV replacement with inactivated poliovirus vaccine (IPV), possible strategies for discontinuing OPV, and the potential for development and licensure of a safe and effective replacement for OPV. ${ }^{12}$

In the framework for national policy makers for OPV using countries, world experts prepared a timetable for OPV cessation in 2005 which can be divided into three distinct periods correlating with the evolution of the major polio risks and risk management strategies. The three distinct periods are as follows: ${ }^{2}$

Phase 1, OPV cessation preparatory phase: this is for three years following the last case of polio by WPV. The risk during the phase is undetected wild transmission assured by optimal AFP surveillance. India is now passing through this phase from 2012-2014.

Phase 2, OPV cessation verification stage: this phase will begin with the simultaneous cessation of OPV and will continue for at least three years thereafter, until verification of the disappearance of Sabin poliovirus strains, absence of cVDPVs. During this phase the any incidence of cVDPVs have to be controlled by type specific monovalent OPV (mOPV).

Phase 3, Post-OPV era: this period will begin with the verification of the disappearance of Sabin-strain polioviruses, as well as the absence of cVDPVs, and will continue indefinitely. Major risks during this period would be the re-introduction of a wild, vaccine derived or Sabin-strain poliovirus. Routine immunization and surveillance should be continued during this period.

\section{Post-eradication strategies}

Resolution 45.17 of the World Health Organization Assembly mandates that only newer vaccines that are cost-effective can be integrated into the national immunization programs of member countries. ${ }^{7}$ In literature, two choices are available, each with four possible scenarios that can be constructed for potential routine vaccination policies. Both the choices and and the possible scenarios are discussed below. ${ }^{3,20}$

Choice I: i) stop all polio vaccination; ii) continue with current vaccination policies (OPV, IPV, or sequential schedule); iii) discontinue OPV, but continue IPV universally; and iv) discontinue OPV, with some countries electing to continue the use IPV. ${ }^{3}$

Choice II: i) continue OPV vaccination; ii) coordinate discontinuation of OPV with or without IPV, depending on national decisions; iii) replace OPV with IPV in all countries before final cessation of polio immunization; and iv) develop new live vaccines that would not cause VAPP and would not be transmissible. ${ }^{21}$

Even after eradication, vaccination can not be stopped abruptly as in choice I.i, since there are chances of poliovirus transmission both as WPV and VDPV, as currently reported from non-endemic countries. Continued use of OPV as in choice II.i will jeopardize the whole world's efforts in polio eradication. At the international level, the global polio control program has used OPV exclusively. While this strategy has succeeded in ending the transmission of WPV, it is being challenged by the fact that, after the global eradication of polio, all cases of paralytic poliomyelitis will be VAPP-associated with the use of OPV. Because live-attenuated poliovirus would be used, it is likely that at any time and anywhere, the conditions may be suitable for VDPVs to acquire the neurovirulence and transmission characteristics of WPV and cause outbreaks. ${ }^{3}$

As explained in the above scenarios, the formulation of a routine vaccination policy for the post-certification era requires that two critical decisions are made: to continue or discontinue vaccination with live attenuated $\mathrm{OPV}$; and, if OPV is discontinued, whether vaccination with IPV is needed.,20

Choice I.ii, sequential use of OPV and IPV: sequential schedule was based on the theory that the development of VAPP was high among non-immune children who received first dose of vaccine. The primary doses are with IPV followed by OPV boosters. But sequential schedule can also result in VAPP, as experienced by the US. After eradication, US tried this option. In January 1997, the Advisory Committee on Immunization Practice recommended the adoption of a sequential IPV/OPV vaccination 
schedule (IPV at 2 and 4 months of age, followed by OPV at 12 to 18 months and again at 4 to 6 years). ${ }^{20}$ Due to occurrence of VAPP on 1 January 2000, the sequential schedule was stopped and changed to the IPV-only schedule. ${ }^{20}$ Both choices I.iii and II.iii suggest discontinuation of OPV, with universal IPV use. The major advantages of scenario iii) are the following: first, it is not associated with VAPP, or the threat of cVDPV or immunodeficient excretors; second, it could maximize a high population immunity.

Choice II.iv suggests the development of new live vaccines that would not cause VAPP and would not be transmissible. This is on the experimental stage. A Sabin-IPV development collaboration among the Netherlands Vaccine Institute, Japan Poliomyelitis Research Institute, and Bio Farma was established in 2005. Sabin-IPV is being developed independently for licensure by the Japan Poliomyelitis Research Institute, by Panacea Biotec of India, and by the Kunming Vaccine Institute in China. ${ }^{21}$ In India we could expect 1 VAPP per 4.2 to 4.6 million doses of OPV. ${ }^{20}$ Considering the threat of VDPVs to polio eradication, the informal WHO meeting urged WHO to develop a strategy to safely discontinue OPV after certification of global eradication. ${ }^{22,23}$ The meeting of the Advisory Committee on polio eradication after estimating the probabilities of VAPPs following the use of OPV after eradication in different countries, led to the decision of stopping the routine use of OPV. ${ }^{23,24}$ The ultimate aim for the post-certification era is to stop OPV safely and effectively, and eventually substitute it with IPV. Further research is urgently needed to answer key scientific and programmatic questions.

\section{Factors against oral poliovirus vaccine use after eradication}

Poliovirus will be eradicated only when OPV use is discontinued and any reintroduction of the virus in the community after eradication will be from continuing use of OPV. ${ }^{12}$ WHO reported that children who have been vaccinated with OPV and are serologically immune can still excrete WPV, and this might contribute to continued transmission despite the high coverage of OPV. ${ }^{9}$ It is a known factor that in tropical countries like India immune responses to OPV are quite unpredictable and erratic, and the vaccine virus take rate is lower in developing countries. A latest published study from India found that after three doses of OPV, the sero conversion rate was only 65 and $63 \%$ for types I and III, respectively, and $96 \%$ for type II. According to most recent estimates from Uttar Pradesh, this would come to a mere $39 \%{ }^{25}$

In the existing epidemiological situation the gut immunity provided by OPV which prevents infection is now undergoing scrutiny. A recent study from India reported that OPV vaccine take is less than expectation, highly seasonal and results in intestinal mucosal immunity that appears to wane significantly within a year of vaccination. ${ }^{24}$ Thus, in areas where faecal oral transmission is high, gut infection with WPV cannot be ruled out. We are having VDPV incidence by type 2 virus reported from various parts of countries even after 14 years of its extinction (1999). This is solely attributed to the use of tOPV which contains type 2 virus. In 2011 India reported seven cases of VDPVs, one of them in a child with congenital immune deficiency in Dhamtari district in Chhattisgarh, and the others in areas with low routine immunization coverage [Udaipur (Rajasthan), Ghaziabad and Badaun (Uttar Pradesh), Barnala (Punjab), Vidisha (Madhya Pradesh) and Jajpur (Orissa) ]. ${ }^{1}$ Similar incidence may happen in the future after eradication if we continue to use OPV with live virus. Hence, the infection may be reintroduced and may cross the borders. In order to avoid cross border reinfection, all countries using OPV should stop OPV use simultaneously in a coordinated manner. ${ }^{17,18}$

\section{Factors favoring the use of inactivated polio vaccine}

Many experts committees associated with WHO advice and policy makers agreed to stop immediately and switch over to use IPV after eradication..$^{19,22,24-27}$ In 2007, the ACPE added to the list of prerequisites the requirement for an affordable IPV that would be appropriate for use in developing countries. The inclusion of IPV in eradication programs requires immediate consideration and the world will need to rely on IPV indefinitely to maintain immunity. ${ }^{18}$ From India field based data were available on the efficacy of IPV better than OPV. In 1985, two IM doses of IPV given to Indian children at the age of 6 weeks at 2 -week-interval or at the age of 8 weeks at 4 -week-interval were having adequate sero-conversion rate against all 3 types of WPV. ${ }^{2}$ In a recently conducted community based randomized controlled trial (RCT) performed at Moradabad, India among infants, the adequate antibodies were reported among $29 \%$ who took mOPV1, $56 \%$ who took intradermal (ID) IPV and 85\% among intramuscular (IM) IPV after 28 days of vaccination. ${ }^{26}$ The only argument raised against the use of IPV was its cost. Considering the priority, resources in terms of man power, money, material, we have to keep the eradication status at any cost to ensure that it will never return in the future. ${ }^{8}$ The current (2010) weighted average purchase prices per dose of vaccine, when purchased by the United Nations Children's Fund (UNICEF), are \$ 0.15 for the trivalent OPV vaccine and approximately US $\$ 3$ for the IPV vaccine..$^{23}$ In order to fully immunize a child of age 5 against polio, the child needs minimum five routine doses of OPV along with five annual doses during national immunization days, a total 10 doses. By substituting with IPV the doses can be reduced to 3 . In an economic evaluation of polio eradication program, experts from the Centre All India Institute of Medical Sciences comment that the direct costs for an intensive pulse polio immunization round was Rs 24.4 per child. In terms of finances and human resources required for pulse polio immunization, we have reached a threshold where new direction and approach is needed to control polio. ${ }^{6}$

The cost of IPV can be reduced by giving fractional doses through ID route. It was an approved scientific fact that the antigens given through ID route are more potent, effective and economic. There is a theoretical advantage of using the dermis as the site of vaccination, including the high density of dendritic cells in the skin compared with the muscle. Intradermal immunization could minimize the inhibitory effect of the passively acquired maternal antibody and thus lead to higher seroconversion rates. ${ }^{21}$

In 1998 Nirmal et al. reported that among Indian new borns aged 6-8 weeks 2 doses of ID IPV at 4- and 8-week-interval produce 90, 80, $98 \%$ and $90,70,97 \%$ seroconvertion for type I, II, III, respectively. ${ }^{27}$ In studies conducted in India, fractional-dose IPV given intradermally resulted in seroconversion rates that were similar to those achieved with the full-dose vaccine. ${ }^{28,29}$ A recently published RCT conducted in a tropical country like Oman showed that fractional doses of IPV vaccine administered intradermally at 2, 4, and 6 months, as compared with full doses of IPV vaccine given intramuscularly on the same schedule, induce similar levels of seroconversion..$^{23}$ A study from Cuba reported less seroconversion with ID IPV with doses at $6,10,14$ weeks. Authors commented that this may be due to genetic variation from India. Half life of maternal derived antibodies range from 29 to 36 days. So, the schedule may be reconsidered according to the local situation. ${ }^{21}$

The cost per infant vaccinated with IPV would be less than $\$ 3$ with the fractional-ID dose vaccine, as compared with $\$ 9$ for the fulldose IM vaccine, a saving of $\$ 6$ per vaccinated infant. ${ }^{23}$ Antigen-sparing techniques such as ID administration could reduce IPV costs significantly, making it more affordable for lowincome countries. ${ }^{18}$ Various ongoing studies by the Global Polio Eradication Initiative on how to make IPV affordable in low income group countries is an ample proof of inevitability of 
its use in later stages of program where there are increase chances for virus transmission. ${ }^{30}$ To reduce the cost along with dose and schedule reduction, other strategies like the use of adjuvants, resulting in a decreased need for antigen, optimization of production processes i.e., increasing cell densities, creating new cell lines, using alternative inactivation agents, can be tried. ${ }^{23}$ The development of an IPV produced from Sabin strains that would be appropriate for production in developing countries can be tried in the future as well. ${ }^{21}$

The Asian country Singapore have got eradication certificate in year $2000 .{ }^{31}$ Given the risk of vaccine-associated paralytic poliomyelitis and circulating vaccine-derived, expert committees advice policy makers to timely consider the replacement of OPV with IPV in national immunisation programs. ${ }^{31}$ An economic study on global polio eradication estimated the costs and made the following comments. ${ }^{32}$ The current cost of routine and intensive OPV immunisation is about US $\$ 2143$ million in the 148 OPV-using countries. Routine use of IPV in these countries should cost US \$ 1246 million. If the current costs of routine and intensive polio immunisation are considered, adopting IPV to replace OPV will not increase the total global cost. Even if the cost of intensive polio immunisation is ignored, cost-effectiveness ratio of adopting IPV remains less than the average gross national income per capita of OPV-using countries. The incremental cost of adopting IPV to replace OPV is relatively low, about US $\$ 1$ per child per year, and most countries should be able to afford this additional cost, ${ }^{32}$ which is applicable to India.

\section{Suitable inactivated polio vac- cine schedule}

On the schedule of IPV, the Indian Association of Pediatrics committee gave two suggestions. ${ }^{33}$ First, sequential as l primary doses of IPV at 6,10 and 14 weeks, followed by two doses of OPV at 6 and 9 months, another dose (booster) of IPV at 15-18 months, and $\mathrm{OPV}$ at 5 years. Due to the risks of VAPP and more number of doses and costs, this sequential dose is not acceptable. Alternatively, two doses of IPV can be used for primary series at 8 and 16 weeks, though this schedule is immunologically superior to EPI schedule and the number of IPV doses is reduced. ${ }^{33}$

As per our stated health policy of self sufficiency of UIP vaccines, with future vision an attempt was made in the 1980s for indigenous manufacture of IPV at public sector. Indian Vaccines Corporation Limited was constituted by Indian Petrochemicals Corporation Ltd and Department of Biotechnology (Government of India) with joint venture of Pasteur
MerieuxSerium and Vaccines, France in 1989. The main objective of the company was to manufacture IPV to be incorporated in the immunizations program of the Government of India. However, IPV was not approved by WHO, subsequently Pasteur MerieuxSerium and Vaccines left the joint venture. In 2008, the entire infrastructure of the company was given on a 30 year lease to $\mathrm{M} / \mathrm{s}$ Reliance Life Sciences Pvt Ltd, for the establishment of a life science research and development Centre at the project site. ${ }^{34,35}$ Thus, the indigenous availability of IPV is now remote in India.

\section{Conclusions}

Though IPV is the appropriate option for India for polio eradication, the Forse cercavi: Ministry of Health and Family Welfare has not made any plan or attempt to get enough IPV or mOPVs stock/supply for the future due to a prejudice against the cost of IPV and a bias towards OPV. India urgently needs to ensure that adequate supplies of vaccines are available for children, so that this eradication adventure does not transform itself into an epidemic disaster. ${ }^{5}$ We need to show urgency and must reject ambiguity, dogmas and prejudices to take some unprecedented decisions. ${ }^{10}$

\section{References}

1. Government of India. Surveillance, at the heart of India's polio success story. Available from: http:/www.searo.who.int/ india/topics/poliomyelitis/surveillance/en/

2. WHO. Cessation of routine oral polio vaccine (OPV) use after global polio eradication. Geneva, Switzerland: World Health Organization; 2005.

3. Sutter RW, Cáceres VM, Mas Lago P. The role of routine polio immunization in the post-certification era. B World Health Organ 2004;82:31-9.

4. WHO. Tracking progress towards global polio eradication, 2010-2011. Geneva, Switzerland: World Health Organization; 2012.

5. Vashisht N, Puliyel J. Polio programme: let us declare victory and move on. Indian $\mathrm{J}$ Med Ethics 2012;9:114-7.

6. Yadav K, Rai SK, Vidushi A, Pandav CS. Intensified pulse polio immunization: time spent and cost incurred at a primary healthcare centre. Natl Med J India 2009;22:13-7.

7. Puliyel JM, Gupta MA, Mathew JL. Polio eradication and the future for other programmes: situation analysis for strategic planning in India. Indian $\mathrm{J}$ Med Res 2007;125:1-4.
8. The Hindu Daily. Two years without polio. Available from: http://www.thehindu.com/ todays-paper/tp-opinion/two-years-without-polio/article4305845.ece

9. WHO. Progress towards eradicating poliomyelitis in India, January 2009October 2010. Geneva, Switzerland: World Health Organization; 2010.

10. Agarwal RK. Polio eradication in India: a tale of science, ethics, dogmas and strategy. Indian J Pediatr 2008;45:349-51.

11. Minor P. Vaccine-derived poliovirus (VDPV): impact on poliomyelitis eradication. Vaccine 2009;27:2649-52.

12. Dowdle WR, De Gourville E, Kew OM, et al. Polio eradication: the OPV paradox. Rev Med Virol 2003;13:277-91.

13. De Silva R, Gunasena S, Ratnayake D, et al. Prevalence of prolonged and chronic poliovirus excretion among persons with primary immune deficiency disorders in Sri Lanka. Vaccine 2012;30:7561-5.

14. Kew OM, Morris-Glasgow V, Landeverde M, et al. Outbreak of poliomyelitis in Hispaniola associated with circulating type 1 vaccine-derived poliovirus. Science 2002;296:356-9.

15. WHO. Paralytic poliomyelitis in Madagascar, 2002. Geneva, Switzerland: World Health Organization; 2002.

16. Centers for Disease Control and Prevention. Acute flaccid paralysis associated with circulating vaccine-derived poliovirus: Philippines. MMWR Morb Mortal Wkly Rep 2001;50:874-5.

17. Jenkins HE, Aylward B, Gasasira A, et al. Implications of a circulating vaccinederived poliovirus in Nigeria. New Engl $\mathbf{J}$ Med 2010;362:2360-9.

18. Modlin JF. The bumpy road to polio eradication. New Engl J Med 2010;25:2346-9.

19. Lahariya C. Global eradication of polio: the case for "finishing the job". Available from: http:/www.who.int/bulletin/volumes/ 85/6/06-037457/en/index.html

20. Xingzhu L, Levin A, Makinen M, Day J. OPV vs IPV: past and future choice of vaccine in the global polio eradication program. Bethesda, MD, USA: The Partners for Health Reformplus Project-U.S. Agency for International Development ed.; 2003.

21. Resik S, Tejeda A, Mas Lago P, et al. Randomized controlled clinical trial of fractional doses of inactivated poliovirus vaccine administered intradermally by needle-free device in Cuba. J Infect Dis 2010;201:1344-52.

22. WHO. Final report of the WHO Informal Consultation on identification and management of vaccine-derived polioviruses, Geneva, 3-5 September 2003. Geneva, Switzerland: World Health Organization; 2003.

23. Mohammed AJ, AlAwaidy S, Bawikar S, et 
al. Fractional doses of inactivated poliovirus vaccine in Oman. New Engl $\mathbf{J}$ Med 2010;362:2351-9.

24. Grassly NC, Jafari H, Bahl S, et al. Waning intestinal immunity after vaccination with oral poliovirus vaccines in India. J Infect Dis 2012;205:1554-61.

25. Paul Y. IPV for OPV primed children. Indian J Pediatr 2012;49:423-4.

26. Estívariz CF, Jafari H, Sutter RW, et al. Immunogenicity of supplemental doses of poliovirus vaccine for children aged 6-9 months in Moradabad, India: a community-based, randomised controlled trial. Lancet Infect Dis 2012;12:128-35.

27. Nirmal S, Cherian T, Samuel BU, et al. Immune response of infants to fractional doses of intradermally administered inactivated poliovirus vaccine. Vaccine
1998;16:928-31.

28. Samuel BU, Cherian MD, Sridharan G, et al. Immune response to intradermally injected inactivated poliovirus vaccine. Lancet 1991;338:343-4.

29. Samuel BU, Cherian MD, Rajasingh J, et al. Immune response of infants to inactivated poliovirus vaccine injected intradermally. Vaccine 1992;10:135.

30. Butcher J. Polio eradication nears the end game. Lancet Neurol 2008;7:292-3.

31. Lee HC, Tay J, Kwok CY, et al. Certification of poliomyelitis eradication in Singapore and the challenges ahead. Ann Acad Med Singap 2012;41:518-28.

32. Khan MM. Economics of polio vaccination in the post-eradication era: should OPVusing countries adopt IPV? Vaccine 2008;26:2034-40.
33. Indian Academy of Pediatrics. Indian Academy of Pediatrics Committee on Immunization (IAPCOI). Consensus recommendations on immunization and iap immunization timetable 2012. Indian J Pediatr 2012;49:549-64.

34. Indian Department of Public Enterprises. Public enterprises survey 2010-2011: Vol-II. Finance service. Vaccine Corporation Ltd. Manesar, Gurgaon, Haryana. Available from: dpe.nic.in/sites/upload_files/dpe /files/survey1011/survey01/volume2/362.pdf

35. The Economic Times. Reliance life science to buy Indian vaccine. Available from: http:/articles.economictimes.indiatimes.c om/2002-07-29/news/27332216_1_ivcolindian-vaccine-corporation-bharat-biotech 